\title{
A Graceful Labeling of Square of Path Graph with Quadratic Complexity Algorithm
}

\author{
FARIS M. TAWEEL ${ }^{1}$, EMAN A. ABUHIJLEH ${ }^{2}$, SHOROUQ ALI $^{3}$ \\ ${ }^{1}$ Software Engineering, Al-Ahliyya Amman University, 19328 Amman, JORDAN \\ ${ }^{2}$ Zarqa University College, Al-Balqa Applied University, Zarqa, JORDAN \\ ${ }^{3}$ Princess Sumaya University for Technology, 11941 Amman, JORDAN
}

\begin{abstract}
A method for relaxed graceful labeling of $P_{n}^{2}$ graphs is presented together with an algorithm designed for labeling these graphs. Graceful labeling is achieved by relaxing the range to $2 m$ and perform the labeling using an algorithm with quadratic complexity $\left(O\left(n^{2}\right)\right)$. The algorithm can be used for labeling an $n \geq 3$, as far as the machine can handle the size of the problem.

Key-Words: - Graceful graph, square of path, algorithm, polynomial complexity, range-relaxed graceful (RRG).
\end{abstract}

Received: April 12, 2021. Revised: August 12, 2021. Accepted: August 22, 2021. Published: September 4, 2021.

\section{Introduction}

Graceful labeling (originally known as $\beta$ valuation) was first introduced by Rosa in 1966 [1]. A graph is graceful if it can be "gracefully numbered." Such numbering can be achieved by labeling all vertices of a graph having $m$ edges with a subset of distinct, nonnegative integers from 0 to $m$, and then, labeling each edge with the absolute difference between the label values of its vertices. The graph becomes a graceful graph if and only if the edge labels run from 1 to $m$. There exists a number of special cases of graceful graphs including, for example: the utility graph $K_{2,3}$ of Gardner[2] and the (generalized) Petersen's graph [3, 4]. It is established that most graphs are not graceful [5].

Graph labeling has applications in many disciplines. It is applied in communication networks, network security, network addressing, channel assignment process, social networks, coding theory, optimal circuits layout, astronomy, radar and graph decomposition problems, as well as in many other area [6]. In particular, in IP networks, graceful labeling of trees has been used in multi-protocol label switching (MPLS) routing platforms [7]. Moreover, Arkut et. al. [8] and Basak [9] proposed an efficient method for managing Internet Protocol (IP) networks using graceful labeling of vertices, that spans caterpillars of the autonomous sub-networks, to assign labels of links in the sub-networks. For further applications on graceful labeling, the reader may refer to Bloom et al.[10] and Sivaraman [11].

In general, the complexity of graceful labeling can be NP-complete [12]. Therefore, many researchers have introduced methods to easing complexity and even to applying it to non-graceful graphs as well. In the sequel of this paper, we present an overview of relevant graceful labeling methods (Section 2); provide theoretical preliminaries (Section 3), analytically show graceful labeling of $P_{n}^{2}$ with a running example (Section 4); then present our method in Section 5, and discuss our results in Section 6. Finally, we conclude with our contributions in Section 7 .

\section{Background}

Asserting about the gracefulness of graphs is mostly given by explicit labeling. For non-graceful graphs, few tools exists for their labeling, and proving gracefulness can be achieved by labeling a graph and then eliminating contradictions among label values until each label is unique.

However, using a deterministic backtracking algorithm, Fang proved that all trees with at most 35 vertices are graceful [13]. This has built on many analytical and computational results of specific classes of trees, including $K_{2}$ graphs, star graphs and Caterpillars of diameter 3 [14]. Also, Zhao proved that all trees with diameter 4 are graceful [15]; Hrnciar and Haviar proved that all trees with diameter 5 are graceful [16]; and Superdock proved that some sub-classes of trees with diameter 6 are graceful [17, 18]. More about graph classes that are graceful and methods for graceful labeling can be found in a survey compiled by Gallian [19].

Relaxing graphs for graceful labeling has been studied since the introduction of graceful graphs themselves, when Rosa introduced variants by relax- 
ing bounds on edges, vertices, or range [1]. Rosa and Siran [20] demonstrated that every tree has an edge-relaxed graceful labeling with at least $\frac{5}{7} m$ different edge labels. Van Bussel [21] and Sivaraman [22] showed that by relaxing bounds on the range of a tree $T$, vertices' labels fall in the range of up to $2 m-\operatorname{diam}(T)$. Adamaszek et al. [23] proved that all trees are almost asymptotically graceful if Rosa conjecture is relaxed such that $\gamma>0$ for all $n>n_{0}(\gamma)$.

In this work, we consider simple, finite and undirected graphs $G$ and focus on $P_{n}^{2}$ where the set of vertices $V\left(P_{n}^{2}\right)=V\left(P_{n}\right)=\left\{v_{0}, v_{1}, \ldots, v_{n}\right\}$ and the set of edges $E\left(P_{n}^{2}\right)=\left\{v_{i} v_{j}|1 \leq| i-j \mid \leq 2\right\}$. Henceforth, the number of edges $m$ for such a graph is $2 n-1$. For this particular graph, we contribute a graceful labeling method by relaxing the range to $2 m$ and perform the labeling using an algorithm with quadratic complexity $\left(O\left(n^{2}\right)\right)$. The algorithm can be used for labeling any $P_{n}^{2}$ graph with $n \geq 3$, as far as the machine can handle the size of the problem.

In the next section, we prelude to our approach with a theoretical basis.

\section{Preliminaries}

A caterpillar and cycle graphs are graceful on constrained cycle values [1]:

Theorem 3.1.

\section{All caterpillars are graceful.}

2. Cycle graph $C_{n}$ is a graceful if and only if $n \equiv$ 0 or $3(\bmod 4)$.

A path graph is a caterpillar with only two end vertices, then for any path graph, it is a graceful graph. Whereas, a complete graph $K_{n}$ is a graceful graph only if it has four or less vertices, Golomb [24]:

Theorem 3.2. A complete graph $K_{n}$ is a graceful graph if and only if $n \leq 4$.

Beutner et al. [25], worked on nearly complete graphs, and established gracefulness by removing 1 , 2 , or 3 edges following the formula $K_{n}-i x$, where $i \in\{1,2,3\}$ :

\section{Theorem 3.3.}

- $K_{n}-x$ is a graceful graph only if $n \leq 5$.

- $K_{n}-2 x$ is a graceful graph only if $n \leq 6$.

- $K_{n}-3 x$ is a graceful graph only if $n \leq 6$.

\section{Gracefulness of $P_{n}^{2}$ for integer}

$$
n \geq 2
$$

For a graceful graph $G$, a search algorithm generates a graceful labeling. If the graph has $m$ edges, then two adjacent vertices must be labeled with 0 and $m$. The $m-1$ label of an edge must has either a vertex with label 1 or label $m-1$, and should be adjacent to a vertex labeled $m$ or 0 , respectively. If a search algorithm follows this pattern in looking for labeled vertices, then it is searching a graceful graph. Let us apply this kind of search algorithm to the graph $P_{n}^{2}$ with a set of vertices $V\left(P_{n}^{2}\right)=\left\{v_{0}, v_{1}, \ldots, v_{n}\right\}$ and a set of edges $E\left(P_{n}^{2}\right)=\left\{v_{i} v_{j}|1 \leq| i-j \mid \leq 2\right\}$. Henceforth, the number of edges $m$ for such a graph is $2 n-1$ where the number of vertices is $q=n+1$.

From theorems 3.2 and 3.3 , one can prove that $P_{n}^{2}$ is a graceful graph with $2 \leq n \leq 4$ as follows:

Proposition 1. A graph $P_{n}^{2}$ with $2 \leq n \leq 4$ is a graceful graph.

\section{Proof of Proposition 1.}

i. For $n=2, P_{2}^{2}$ is isomorphic to $K_{3}$. Hence, it is graceful by Theorem 3.2

ii. For $n=3, P_{3}^{2}$ is isomorphic to $K_{4}-x$. Hence, it is graceful by Theorem 3.3

iii. For $n=4, P_{4}^{2}$ is isomorphic to $K_{5}-3 x$. Hence, it is graceful by Theorem 3.3

We have shown that $P_{n}^{2}$ is a graceful graph using an algorithm but with exponential complexity For a number of $P_{n}^{2}$ graphs, Table 1 presents possible solutions for each graph, showing the maximum number of solutions and time in seconds for identifying all solutions up to $n$ equals 14 . Considering the limited platform resources, we have altered the algorithm to find one possible solution for graphs with vertices greater than $n$ equals 14 . The platform was capable of generating partial sets of the solutions for graphs with $n$ equals 22 . In the Table 11, the number of solutions super-scripted with a star $\left(^{*}\right)$ are subsets of the solutions and henceforth the time is much smaller than in previous results. The platform used for this calcula-

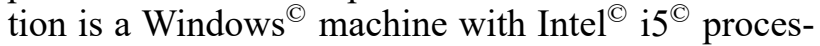
sor and 16GB of RAM. Furthermore, the algorithm consists of a depth-first search algorithm (DFS) and a breadth-first search algorithm (BFS) [26]. It generates all possible labels of $P_{n}^{2}$ for $n \geq 3$; agreeing with Kang2] et al. [27].

\footnotetext{
${ }^{1}$ Only results of this algorithm are presented in this context.

${ }^{2}$ Link to the journal or the full article can not be established, as stated at https://mathscinet.ams.org/mathscinet-getitem?mr=1418071
} 


\begin{tabular}{|c|l|l|l|}
\hline No. of Vertices & Labels of vertices & $\begin{array}{l}\text { No. of Solu- } \\
\text { tions }\end{array}$ & Time (sec.) \\
\hline$=n+1$ & (sample) & 16 & 1.714700 \\
\hline 4 & $0,5,4,2$ & 121 & 4.126300 \\
\hline 7 & $0,11,10,2,5,9,3$ & 164 & 3.835200 \\
\hline 8 & $0,13,12,3,1,9,6,2$ & 389 & 13.498900 \\
\hline 9 & $0,15,1,11,14,2,7,13,5$ & 909 & 7.764800 \\
\hline 10 & $0,17,16,2,12,15,3,8,14,6$ & 2010 & 14.951700 \\
\hline 11 & $0,19,1,8,18,2,4,17,13,5,10$ & 3539 & 20.834700 \\
\hline 12 & $0,21,1,16,20,4,2,11,19,5,8,18$ & 8300 & 207.371900 \\
\hline 13 & $0,23,1,3,22,8,4,21,11,5,20,17,9$ & $16^{*}$ & 0.886000 \\
\hline 16 & $0,29,1,3,28,17,4,27,8,5,26,11,6,23,14,7$ & $20^{*}$ & 0.661200 \\
\hline 19 & $35,0,1,33,31,2,20,30,3,26,29,4,13,28,7,15,27,8,22$ & $20^{*}$ & 0.665900 \\
\hline 20 & $0,37,1,3,36,31,4,35,14,5,34,23,8,11,33,25,9,32,26,13$ & & \\
\hline
\end{tabular}

Table 1: Graceful labeling of $P_{n}^{2}$ using DFS-BDS algorithm with exponential complexity.

In the next section, a range-relaxed graceful (RRG) algorithm is designed for generating graceful labeling of $P_{n}^{2}$ with $n \geq 3$. This algorithm assumes that the graph's range is relaxed to $2 \mathrm{~m}$. In some cases, the range needs to be relaxed by $3 m$ (see Observation 5.2).

\section{Range-Relaxed Graceful labeling of $P_{n}^{2}$}

The main contribution of this work is a graceful labeling of a $P_{n}^{2}$ graph with polynomial complexity. Our approach is achieved by relaxing the graph range from $m$ to $2 m$. In this section, we manually label $P_{7}^{2}$ and then specify our algorithm for solving the general problem of graceful labeling of $P_{n}^{2}$ graphs.

\subsection{Running Example}

To demonstrate, let us derive a $P_{7}^{2}$ graph from a $P_{7}$ graph shown in Fig. 11. The set of vertices of both graphs is $\left\{v_{0}, v_{1}, v_{2}, v_{3}, v_{4}, v_{5}, v_{6}, v_{7}\right\}$, where $v_{i} v_{j} \in$ $E\left(P_{7}^{2}\right)$ if and only if $1 \leq|i-j| \leq 2$. We organize our

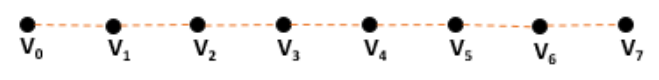

Figure 1: A simple $P_{7}^{2}$ graph.

solution in steps starting with Step 0. In this step, and for the ease of clarity, we connect by a directed, bold arrow every two vertices of $P_{7}$ that are separated by a path of length 2 (Fig 2a), and re-organize it as shown in Fig $2 b$ The dashed line in Fig.2 represents the path connecting vertices of the original graph.

In Step 1, we label vertices with even index using the following rule:

$$
f_{e}\left(v_{i}\right)= \begin{cases}\frac{i}{4} & ; i \equiv 0(\bmod 4) \\ m-\left(\frac{i-2}{4}\right) & ; i \equiv 2(\bmod 4)\end{cases}
$$

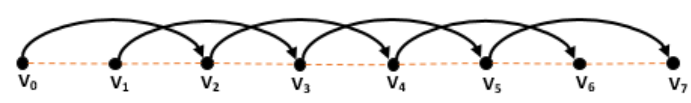

(a) Connected vertices

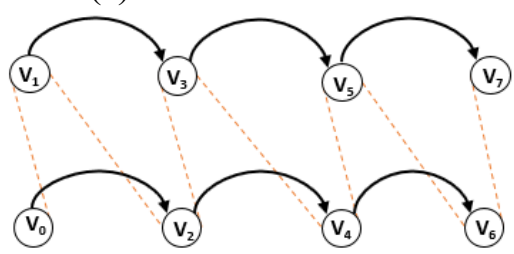

(b) Reorganized graph of connected vertices

Figure 2: Preparation of $P_{7}^{2}$ for labeling (Step 0)

The result of applying $f_{e}\left(v_{i}\right)$ is shown in Fig $3 \mathrm{a}$ and Fig. $3 \mathrm{~b}$, where $g$ is the set of edge labels evaluated by the formula $g\left(v_{i} v_{j}\right)=\left|f\left(v_{i}\right)-f\left(v_{j}\right)\right|$.

In Step 2, we continue by labeling odd vertices using the following rule:

$$
f_{o}\left(v_{i}\right)= \begin{cases}\left\lceil\frac{n}{2}\right\rceil & ; i=1 \\ 2^{\left(\frac{i-1}{2}\right)}+f\left(v_{i-2}\right) & ; i \neq 1 \wedge \\ & i \equiv \\ & 1 \bmod 2\end{cases}
$$

We proceed with this step by labeling vertex by vertex, and when evaluating the label of each vertex, we check if the corresponding edge labels are already in $g$. If all the new edge labels are unique and not in $g$, we continue with next vertex. Otherwise, we have a conflict. In this example, when labeling $v_{5}$ by $f_{o}\left(v_{5}\right)$, the $g$ label of the edge connecting $v_{0}$ and $v_{1}$ has the same value of the edge label connecting $v_{3}$ and $v_{5}(4$ is already a member of $g$ as shown Fig $4 \mathrm{~b}$; highlighted by the arrow).

In this part of Step 2, let us define a set $S$ of integers $i$ such that $0 \leq i \leq 2 m$, where $2 m=26$. We are interested in a sorted set $S^{\prime} \subseteq S$ such that 


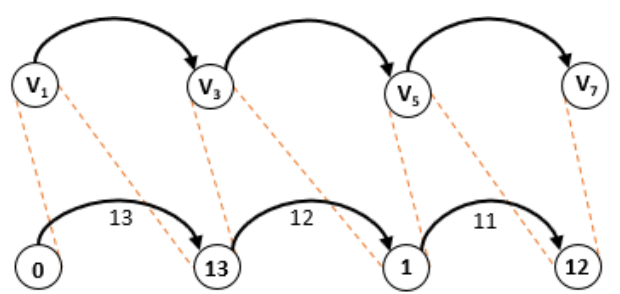

(a) Starting graceful labeling of the graph.

\begin{tabular}{ccc}
\hline$v_{i}$ & $\mathbf{f}$ & $\mathbf{g}$ \\
\hline$v_{0}$ & 0 & - \\
$v_{2}$ & 13 & $g=13$ \\
$v_{4}$ & 1 & $g=12$ \\
$v_{6}$ & 12 & $g=11$ \\
\hline
\end{tabular}

(b) Table of labeling results.

Figure 3: Step 1 showing initial labeling values.

$S^{\prime}=S-f$, where $f=\operatorname{Im}\left(f_{e}\right) \cup \operatorname{Im}\left(f_{o}\right)$. We start by assigning $f\left(v_{5}\right)$ the first value in $S^{\prime}$. If the resulting edge labels for this value are not in the range of $g$, then we append the value from $S^{\prime}$ in the set $f$ and append the values evaluated using this label value in the set $g$. Otherwise, we repeat this step by selecting the next smallest value in $S^{\prime}$ until a value is found that produces edge labels not in $g$, and so forth. This step has been performed on $v_{5}$ and $v_{7}$ leading to the results summarized in Fig.5. In this particular example, the order of complexity is $O(n)$.

\subsection{An RRG Algorithm}

Manually labeling $P_{7}^{2}$ in Section 5.1 highlighted the main steps of an RRG algorithm for graceful labeling of $P_{n}^{2}$ graphs. These steps are:

- Step 0: Initialization of variables and sets.

- Step 1: Labeling of even-indexed vertices.

- Step 2: Piece-wise labeling of the remaining odd-indexed vertices.

In writing a specification for this algorithm, we have much of the notation from refinement calculus [28]. But also, included some imperative code and textual comments. The algorithm has been refined to $C++$ code and compiled on a Windows 10 platform.

In Line 0.4 of Specification 1, we assume that $S^{\prime}$ is sorted in ascending order; $e_{0} \leq e_{1} \leq \ldots$, and in Line $0.6, n$ ? denotes a data input of an integer greater or equals to three. We also define an operator $\lambda$ such $\lambda_{k} S^{\prime}$ returns the $k^{t h}$ element $e_{k} \in S^{\prime}$; i.e., $e_{k}:=\lambda_{k} S^{\prime}$.

Line 1.1 of Specification 2 asserts that $n<3$ or aborts the algorithm, and lines 1.2 and 1.3 assigns la-

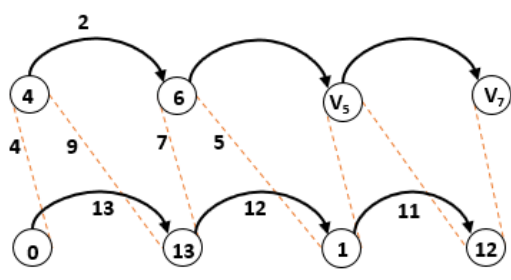

(a) Graceful labeling fails at $v_{5}$.

\begin{tabular}{ccc}
\hline $\mathbf{v}_{\mathbf{i}}$ & $\mathbf{f}$ & \multicolumn{1}{c}{$\mathbf{g}$} \\
\hline \multirow{3}{*}{$\mathrm{v}_{1}$} & 4 & $\mathrm{~g}_{0}=9$ \\
& & $\mathrm{~g}_{1}=\underline{4}$ \\
& & $\mathrm{~g}_{2}=2$ \\
$\mathrm{v}_{3}$ & 6 & $\mathrm{~g}_{1}=7$ \\
& & $\mathrm{~g}_{0}=5$ \\
& & $\mathrm{~g}_{2}=4$ \\
$\mathrm{v}_{5}$ & 10 & $\mathrm{~g}_{1}=$ \\
& & $\mathrm{g}_{0}=$ \\
& &
\end{tabular}

$v_{7}$

(b) $g_{2}=4$ conflicts with $g_{1}=4$.

Figure 4: Step 2 shows how labeling has failed when $g_{1}=4$ and $g_{2}=4$.

bels to even-indexed vertices and edges connecting these vertices.

The algorithm generates graceful labeling of a $P_{n}^{2}$ graph whose range is relaxed to $2 m$ for any $n \geq 3$, with quadratic complexity $O\left(n^{2}\right)$. This contribution is formally stated below with a proof.

Theorem 5.1. The labeling algorithm generates a relaxed graceful labeling for $P_{n}^{2}$ graph with a relaxed range $2 m$ where $n \geq 3$.

\section{Proof of Theorem 4.}

Step 0: Definitions of main variables and sets for this algorithm are given in Specification 1.

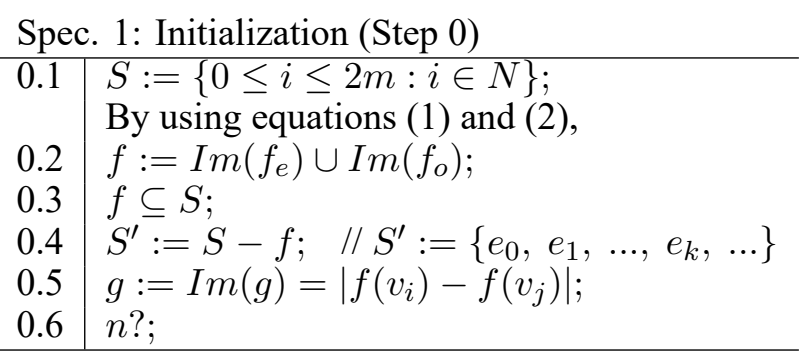

Spec. 2: Labeling even-indexed vertices (Step 1)

\begin{tabular}{|c|c|}
\hline 1.1 & if $n<3 \rightarrow S K I P:$ fi; \\
\hline 1.2 & $i:=0 ;$ do $i \leq \frac{n}{2} \rightarrow f:=\left\{f_{e}\left(v_{2 i}\right)\right\} \cup f ;$ od $;$ \\
\hline 1.3 & $\begin{aligned} i:=0 ; \text { do } i & <\frac{\bar{n}}{2} \rightarrow \\
& g:=\left\{g\left(v_{2 i} v_{(2(i+1))}\right)\right\} \cup g ; \text { od } ;\end{aligned}$ \\
\hline
\end{tabular}


Spec. 3.1: Labeling odd-indexed vertices (Step 2.1)

\begin{tabular}{|c|c|}
\hline 2.01 & $i:=0 ;$ do $i \leq \frac{n-1}{2} \rightarrow$ \\
\hline 2.02 & $a f:=f_{o}\left(v_{2 i+1}^{2}\right)$ \\
\hline 2.03 & $i f(2 i+1)=1 \rightarrow$ \\
\hline 2.04 & $\begin{array}{l}\text { if }\left\{\left|f_{e}\left(v_{2 i}\right)-f_{o}\left(v_{2 i+1}\right)\right|\right\} \subseteq g \vee \\
\quad\left\{\left|f_{o}\left(v_{2 i+1}\right)-f_{e}\left(v_{2(i+1)}\right)\right|\right\} \subseteq g \rightarrow\end{array}$ \\
\hline 2.05 & $k:=0 ;$ do $k \leq\left|S^{\prime}\right| \rightarrow e_{k}:=\bar{\lambda}_{k} S^{\prime}$ \\
\hline 2.06 & $\begin{array}{l}i f\left\{\left|f_{e}\left(v_{2 i}\right)-e_{k}\right|\right\} \not g g \wedge \\
\left\{\left|e_{k}-f_{e}\left(v_{2(i+1)}\right)\right|\right\} \nsubseteq \nsubseteq g \wedge\end{array}$ \\
\hline 2.07 & $\mid \begin{array}{l}\left|f_{e}\left(v_{2 i}\right)-f_{o}\left(v_{2 i+1}\right)\right| \neq \\
\left|f_{o}\left(v_{2 i+1}\right)-f_{e}\left(v_{2(i+1)}\right)\right| \rightarrow\end{array}$ \\
\hline 2.08 & $\begin{aligned} g & :=\left\{\left|f_{e}\left(v_{2 i}\right)-e_{k}\right|\right. \\
& \left.\left|e_{k}-f_{e}\left(v_{2(i+1)}\right)\right|\right\} \cup g\end{aligned}$ \\
\hline 2.09 & $f:=f \cup\left\{e_{k}\right\}$ \\
\hline 2.10 & break;//break loop \\
\hline 2.11 & $f i$; \\
\hline 2.12 & od; \\
\hline 2.13 & else \\
\hline 2.14 & $g:=g \cup\left\{\left|f_{e}\left(v_{2 i}\right)-f_{o}\left(v_{2 i+1}\right)\right|\right.$, \\
\hline 2.15 & $\left.\left|f_{o}\left(v_{2 i+1}\right)-f_{e}\left(v_{2(i+1)}\right)\right|\right\}$ \\
\hline 2.16 & $f:=f \cup\{a f\}$ \\
\hline 17 & $f i$ \\
\hline
\end{tabular}

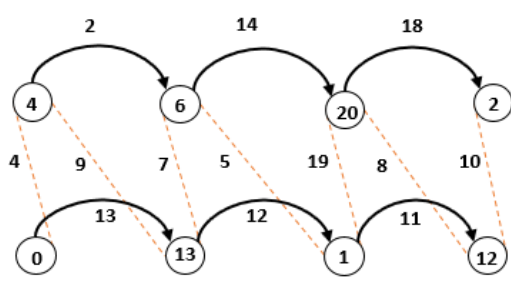

(a) Graph fully labeled.

\begin{tabular}{ccl}
\hline v & $\mathbf{f}$ & \multicolumn{1}{c}{$\mathbf{g}$} \\
\hline \multirow{2}{*}{$\mathrm{v}_{1}$} & 4 & $\mathrm{~g}_{0}=9$ \\
& & $\mathrm{~g}_{1}=4$ \\
& & $\mathrm{~g}_{2}=2$ \\
$\mathrm{v}_{3}$ & 6 & $\mathrm{~g}_{1}=7$ \\
& & $\mathrm{~g}_{0}=5$ \\
& & $\mathrm{~g}_{2}=14$ \\
$\mathrm{v}_{5}$ & 20 & $\mathrm{~g}_{1}=19$ \\
& & $\mathrm{~g}_{0}=8$ \\
& & $\mathrm{~g}_{2}=18$ \\
$\mathrm{v}_{7}$ & 2 & $\mathrm{~g}_{1}=10$ \\
& & $\mathrm{~g}_{0}=$ skip $\mathrm{i}=\mathrm{n}$ \\
\hline
\end{tabular}

(b) Table of the labeling results.

Figure 5: Final graceful labeling of $P_{n}^{2}$.

Step 1: Evaluating $f_{e}\left(v_{i}\right)$ of even indices, updating $S^{\prime}$ (i.e. remove $f_{e}\left(v_{i}\right)$ from $S$ ) and appends edge labels to set $g$.

Spec. 3.2: Labeling odd-indexed vertices (Step 2.2) Step 2: Evaluating $f_{o}\left(v_{i}\right)$ of odd indices, and testing in

$2.18 \quad$ else if $(2 i+1)<n$

$2.19 \quad$ if $\left\{\left|f_{o}\left(v_{2 i-1}\right)-f_{o}\left(v_{2 i+1}\right)\right|\right\} \subseteq g$

$2.20 \quad \vee\left\{\left|f_{o}\left(v_{2 i+1}\right)-f_{e}\left(v_{2 i}\right)\right|\right\} \subseteq g$

$2.21 \quad \vee\left\{\left|f_{o}\left(v_{2 i+1}\right)-f_{e}\left(v_{2(i+1)}\right)\right|\right\} \subseteq g \rightarrow$

$2.22 \quad k:=0 ;$ do $k \leq\left|S^{\prime}\right| \rightarrow e_{k}:=\lambda_{k} S^{\prime}$

$2.23 \quad$ if $\left\{\left|f_{o}\left(v_{2 i-1}\right)-e_{k}\right|\right\} \nsubseteq g$

$2.24 \wedge\left\{\left|e_{k}-f_{e}\left(v_{2 i}\right)\right|\right\} \nsubseteq \subseteq g$

2.25

2.26

2.27

2.28

2.29

2.30

2.31

2.32

2.33

2.34

2.35

2.36

2.37

2.38

2.39 $\wedge\left\{\left|e_{k}-f_{e}\left(v_{2(i+1)}\right)\right|\right\} \nsubseteq g$

$/ /$ elements of a set are unique

$\wedge \mid\left\{\left|f_{o}\left(v_{2 i-1}\right)-e_{k}\right|,\left|e_{k}-f_{e}\left(v_{2 i}\right)\right|\right.$, $\left.\left|e_{k}-f_{e}\left(v_{2(i+1)}\right)\right|\right\} \mid=3 \rightarrow$

$g:=g \cup\left\{\left|f_{o}\left(v_{2 i-1}\right)-e_{k}\right|\right.$,

$\left|e_{k}-f_{e}\left(v_{2 i}\right)\right|$,

$\left.\left|e_{k}-f_{e}\left(v_{2 i+2}\right)\right|\right\} ;$

$f:=f \cup\left\{e_{k}\right\}$;

break;//break loop

$f i$

$o d ;$

else

$$
\begin{aligned}
g:=g \cup\{\mid & f_{o}\left(v_{2 i-1}\right)-f_{o}\left(v_{2 i+1}\right) \mid, \\
& \left|f_{o}\left(v_{2 i+1}\right)-f_{e}\left(v_{2 i}\right)\right|, \\
\mid & \left.f_{o}\left(v_{2 i+1}\right)-f_{e}\left(v_{2(i+1)}\right) \mid\right\} ;
\end{aligned}
$$

$f:=f \cup\{a f\}$;

\section{each step if the following conditions hold:}

Spec. 3.3: Labeling odd-indexed vertices (Step 2.3)

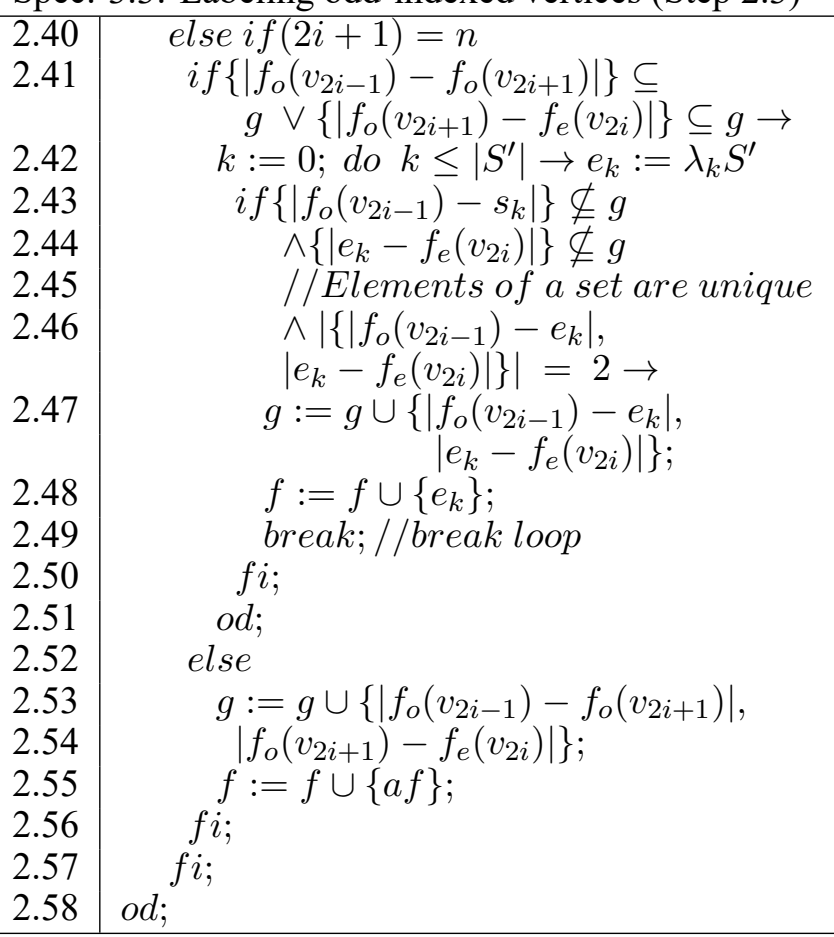


1. Vertex label is unique (set $f$ ).

2. Edge labels are unique (set $g$ ).

Then, update $S^{\prime}$ and append edge labels to the set $g$. If in this step a conflict occurs, iterate in $S^{\prime}$ to calculate labels that satisfy the graceful labeling property. It is important to note that by contradiction, one can prove that $f_{e} \cap f_{o}=\phi, f_{e}$ and $f_{o}$ are one-to-one functions.

According to the previous theorem, we have the following observations:

The labeling algorithm has a relaxed range set, denoted by $S=\{0,1, \ldots, 2 m\}$, where $S^{\prime}$ is the updated, sorted set of $S$.

The labeling algorithm works at a relaxed range of $2 m$ for most cases. Some cases have relaxed range up to $2.7 m$, e.g. $n \in\{20,39,41,42,57,77,88\}$.

Based on the algorithm we have presented in this work, we propose the following:

Proposition 2. The complexity of the relaxed labeling algorithm of $P_{n}^{2}$, with a relaxed range of $2 m$ and $n \geq$ 3 , is of the order of $O\left(n^{2}\right)$ :

\section{Proof of Proposition 3.}

1. Step one runs $\left\lceil\frac{n+1}{2}\right\rceil$ times, while index label is even.

2. Step two runs $\left(\left\lfloor\frac{n+1}{2}\right\rfloor\right)\left((4 n-2)-\left\lceil\frac{n+1}{2}\right\rceil\right)$ for odd index labels.

Consequently, the complexity function can be expressed as follows:

$$
h(n)=\left\lceil\frac{n+1}{2}\right\rceil+\left\lfloor\frac{n+1}{2}\right\rfloor\left((4 n-2)-\left\lceil\frac{n+1}{2}\right\rceil\right)
$$

Hence, the complexity is of the order of $O\left(n^{2}\right)$.

\section{Discussion}

Many researchers have investigated graceful labeling of different types of graphs, with or without relaxation. However, as far as our work is concerned, no other research has directly investigated graceful labeling of $P_{n}^{2}$ graphs. But, it deserves mentioning few works that have had addressed graceful labeling by analytical means or computational methods.
Recently, Adamaszek et al. [23] provided an algorithm to find a graceful labeling of a tree by proposing a relaxation of Rosa conjecture [1]. The proposed relaxation based on assuming $\gamma>0$ and for all $n>n_{0}(\gamma)$, an upper bound $O_{\gamma}\left(\frac{n}{\log n}\right)$ set on the tree degree, and choosing vertex labels from $\{1,2$, $\ldots .,\lceil(1+\gamma) n\rceil\}$. The algorithm finds a graceful labeling under these conditions, but the complexity of the algorithm has not been quantified. The method and its algorithm processes trees which are bipartite graphs. Our method and algorithm find graceful labeling of square of path graphs with complexity of $O\left(n^{2}\right)$.

In [13], without relaxation, a hybrid algorithm is used to perform graceful labeling of a tree graph. The algorithm implements labeling in two search stages: (i) a deterministic backtracking stage and (ii) a probabilistic stage. The first stage is an optimized, deterministic algorithm based on Horton's proposal of randomized backtracking search for graceful labeling [29]. However, the number of backtracks in this algorithm can grow exponentially, henceforth a threshold has been added on the number of backtracks. The probabilistic stage algorithm is based on hill-climbing tabu search proposed by [31] combined with ideas from simulated annealing from combinatorial optimization. The resulted hybrid algorithm, applies the first stage, and if it fails in finding a graceful labeling, the second stage is started. It has been observed that the deterministic backtracking search is sufficient to label $99.9 \%$ of trees. This algorithm computes graceful labeling of trees of order up to 35 vertices, improving over previous works [29,30,31], but the complexity of the algorithm has not been explicitly specified.

Beutner et al. investigated nearly complete graphs focused at $K_{n}$ and removed 1,2, or 3 edges [25]. Without relaxation, graphs $K_{4}-x$ and $K_{5}-3 x$ are isomorphic to $P_{3}^{2}$ and $P_{4}^{2}$ respectively. That is, we have analytical proof of gracefulness of the later graphs up to $n=4$. Furthermore, authors proved gracefulness of tripartite $K_{1, a, b}$ and $K_{2, a, b}$ that are not isomorphic to the tripartite $P_{n}^{2}$ when $n \geq 4$.

In a relevant work to ours, Redl has used a constraint programming approach to show that the general graph $P(n, k)$ is graceful for $5 \leq n \leq 10$ [3] and Shao et al.[4] has managed to label the graph for $n \leq 75$. Both used algorithms to generate labeling without calculating the complexity, but Shao algorithm is faster than Redl's.

In the context of range relaxation, Bussel (2002) and Sethuraman (2017) relaxed the range of a tree graph up to $2 m$ - diameter, where $m$ is the number of edges [21, 22]. Sethuraman has achieved labeling with a smaller range but without explicit mention of complexity. While Bussel investigated graceful labeling of graphs with larger size with a cost of expo- 


\begin{tabular}{|c|c|c|c|c|}
\hline \multirow[b]{2}{*}{$\begin{array}{l}\text { No. of Vertices } \\
\qquad q=n+1 \\
\end{array}$} & \multicolumn{2}{|c|}{ Exponential Algorithm on range $m$} & \multicolumn{2}{|c|}{ Polynomial algorithm on range $2 m$} \\
\hline & $\begin{array}{l}\text { Labels of vertices } \\
\text { (sample) }\end{array}$ & $\begin{array}{l}\text { Time } \\
\text { (sec.) }\end{array}$ & Labels of vertices & $\begin{array}{l}\text { Time } \\
\text { (sec.) }\end{array}$ \\
\hline$\overline{4}$ & $0,5,4,2$ & 1.714700 & $\overline{00,2,5,1}$ & 0.04155 \\
\hline 7 & $0,11,10,2,5,9,3$ & 4.126300 & $0,3,11,5,1,17,10$ & 0.020720 \\
\hline 8 & $\begin{array}{l}0,13,12,3,1,9,6, \\
2\end{array}$ & 3.835200 & $\begin{array}{l}0,4,13,6,1,20 \\
12,2\end{array}$ & 0.019700 \\
\hline 9 & $\begin{array}{l}0,15,1,11,14,2 \\
7,13,5\end{array}$ & 13.498900 & $\begin{array}{l}0,4,15,6,1,7,14 \\
24,2\end{array}$ & 0.044971 \\
\hline 10 & $\begin{array}{l}0,17,16,2,12,15 \\
3,8,14,6\end{array}$ & 7.764800 & $\begin{array}{l}0,5,17,7,1,8,16 \\
27,2,3\end{array}$ & 0.040651 \\
\hline 11 & $\begin{array}{l}0,19,1,8,18,2,4 \\
17,13,5,10\end{array}$ & 14.951700 & $\begin{array}{l}0,5,19,7,1,11 \\
18,31,2,28,17\end{array}$ & 0.065612 \\
\hline 12 & $\begin{array}{l}0,21,1,16,20,4 \\
2,11,19,5,8,18\end{array}$ & 20.834700 & $\begin{array}{l}0,6,21,8,1,12 \\
20,34,2,3,19,4\end{array}$ & 0.057235 \\
\hline 13 & $\begin{array}{l}0,23,1,3,22,8,4 \\
21,11,5,20,17,9\end{array}$ & 207.371900 & $\begin{array}{l}0,6,23,8,1,12, \\
22,36,2,5,21,30 \\
3\end{array}$ & 0.060580 \\
\hline 16 & $\begin{array}{l}0,29,1,3,28,17 \\
4,27,8,5,26,11 \\
6,23,14,7\end{array}$ & $0.886000^{*}$ & $\begin{array}{l}0,8,29,10,1,14 \\
28,13,2,7,27,37 \\
3,19,26,4\end{array}$ & 0.083707 \\
\hline 19 & $\begin{array}{l}35,0,1,33,31,2 \\
20,30,3,26,29,4 \\
13,28,7,15,27,8 \\
22\end{array}$ & $0.661200^{*}$ & $\begin{array}{l}0,9,35,11,1,15 \\
34,23,2,39,33, \\
16,3,52,32,7,4 \\
46,31\end{array}$ & 0.113140 \\
\hline 20 & $\begin{array}{l}0,37,1,3,36,31, \\
4,35,14,5,34,23, \\
8,11,33,25,9,32, \\
26,13\end{array}$ & $0.665900^{*}$ & $\begin{array}{l}0,10,37,12,1,16 \\
36,24,2,40,35 \\
17,3,43,34,62,4 \\
75,33,5\end{array}$ & 0.287005 \\
\hline
\end{tabular}

Table 2: Contrasting results of an exponential algorithm vs the relaxed algorithm for a number of $P_{n}^{2}$ graphs. Star $\left({ }^{*}\right)$ in the Time column indicates time spent in searching for subset of solutions.

nential complexity. Compared to our work, authors of these works have achieved labeling with a smaller range. However, they did not calculate or comment on the complexity of their algorithm.

We have performed graceful labeling of a graph by relaxing the range to $2 m$ achieving quadratic complexity.

Compared to our work, [3, 4, 25] and [29, 30, 31] performed graceful labeling of graphs with restriction on the number of vertices and without explicit mention of complexity, though we believe their complexity is exponential. In other works, range was relaxed to at most $2 m$ but without computing complexity as well [21, 22].

Finally, Montgomery et al. [32] have recently proved Ringel's conjecture which conjectures a graceful labeling of many special classes of trees including caterpillars, trees with 4 leaves, Firecrackers, diameter 5 trees, symmetrical trees, trees with 35 vertices, and olive trees. But their proof is equivalent to $\rho$-graceful which is weaker than graceful labeling.
Ringel's conjecture is weaker than graceful labeling conjecture that we have achieved in our work.

We have developed the specification of our algorithm in $\mathrm{C}++$ (Section 5.2). On an i5 machine with 16GB RAM, we tested the algorithm with graph sizes up to $n=500,0000$, and it took 36 hours to finish such maximum on this platform. A sample run of the exponential and the relaxed algorithms is summarized in Table2.

\section{Conclusion}

The main contribution of this work is a graceful labeling of square of path graphs $\left(P_{n}^{2}\right)$ with a polynomial complexity of the order of $O\left(n^{2}\right)$. Our approach is achieved by relaxing the range from $m$ to $2 m$. We applied a labeling algorithm that finds labels for evenindexed vertices, and then piece-wise labeling the remaining odd-indexed vertices. The steps of the algorithm has been demonstrated by a small size graph $\left(P_{n}^{2}\right)$ and it has been speciefied using refinement calculus notation. 
The algorithm has been implemented in $\mathrm{C}++$ and run on a Windows ${ }^{\odot}$ machine with Intel $^{\odot}{ } 5^{\odot}$ and 16GB RAM. Results has confirmed our calculated complexity which is of the order of $O\left(n^{2}\right)$.

\section{References:}

[1] A. Rosa, On certain valuations of the vertices of a graph, in: In Proceedings of the International Symposium of the Theory of Graphs, International Computing Centre, Rome, July, 1966.

[2] Golomb, Graceful Graphs, W. H. Freeman, Ch. 15, pp. 152-165.

[3] T. Redl, Graceful graphs and graceful labelings: Two mathematical formulations and some other new results, Congr. Numer. 164 (2003) 17-31.

[4] Vesel, Aleksander and Shao, Zehui and Deng, Fei and $\mathrm{Li}$, Zepeng, Graceful labelings of the generalized petersen graphs, Communications in Combinatorics and Optimization 2 (2) (2017) 149-159. doi:DOI:10.22049/CCO.2017.25918.1055.

[5] R. L. Grahams, N. J. A. Sloane, On additive bases and harmonious graphs, SIAM Journal on Algebriac and Discrete Methods 1 (1980) 382-404.

[6] M. Sutton, Summable graph labelings and their applications, Ph.D. thesis, School of Management, University of Newvastle (2001).

[7] I. C. Arkut, R. C. Arkut, N. Ghani, Graceful label numbering in optical MPLS networks, Vol. 4233 of Society of Photo-Optical Instrumentation Engineers (SPIE) Conference Series, 2000, pp. 1-8. doi:10.1117/12.401809.

[8] R. C. A. I. C. Arkut, A. Basak, "topology constrained label switching for multi-cast routing", in: Eighth IEEE Symposium on Computers and Communications, ISCC, Kemer-Antalya, Turkey, 2003, pp. 453-459.

[9] A. Basak, "MPLS multi-casting using caterpillars and a graceful labeling scheme", in: Eighth International Conference on Information Visualization, Vol. IV, IEEE, London, UK, 2004, pp. 382387.

[10] G. S. Bloom, S. W. Golomb, Applications of numbered undirected graphs, Proceedings of the IEEE 65 (4) (1977) 562-570.

[11] R. Sivaraman, Graceful graphs and its applications, International Journal of Current Research 8 (11) (2016) 41062-41067.

[12] S. Skiena, The Algorithm Design Manual, Second Edition, Springer, 2008.
[13] W. Fang, A computational approach to the graceful tree conjecture, CoRR abs/1003.3045, 2010. URL http://arxiv.org/abs/1003.3045

[14] R. M. Zhou, Graceful labeling of graphs, Master's thesis, UFRJ/COPPE, Riode Janeiro (2016).

[15] S.-L. Zhao, All trees of diameter four are graceful, Annals of the New York Academy of Sciences 576 (1) (2006) 700706. doi:10.1111/j.1749-6632. 1989.tb16451.x. URL https://doi.org/10.1111/j.17496632.1989.tb16451.x

[16] P. Hrnciar, A. Haviar, All trees of diameter fi ve are graceful, Discrete Mathematics $233 \quad(1-3) \quad(2001)$ 133-150. doi:10.1016/S0012-365X(00)00233-8. URL https://doi.org/10.1016/S0012-365X(00)00233-8

[17] M. C. Superdock, The graceful tree conjecture: A class of graceful diameter- 6 trees, 2013.

[18] M. Superdock, New graceful diameter- 6 trees by transfers, 2014.

[19] J. Gallian, A dynamic survey of graph labeling, Electronic Journal of Combinatorics 1 (DynamicSurveys) (2019) 1-535.

[20] A. Rosa, J. Sirán, Bipartite labelings of trees and the gracesize, Graph Theory 19 (2) (1995) 201-215. doi:10.1002/jgt.3190190207. URL https://doi.org/10.1002/jgt.3190190207

[21] F. V. Bussel, Relaxed graceful labelings of trees the electronic journal of combinatorics 9 (R4).

[22] G. Sethuraman, P. Ragukumar, P. J. Slater, Any tree with $m$ edges can be embedded in a graceful tree with less than $4 \mathrm{~m}$ edges and in a graceful planar graph, Discrete Math. 340 (2) (2017) 96-106. doi:10.1016/j.disc. 2016.07.009. URL https://doi.org/10.1016/j.disc.2016.07.009

[23] A. Adamaszek, P. Allen, C. Grosu, J. Hladky, Almost all Trees are Almost Graceful, arXiv:1608.01577v6 [math.CO] 24 Sep 2019.

[24] S. W. Golomb, How to number a graph, in: Graph Theory and Computing, Academic Press, New York, USA, 1972, pp. 23-37.

[25] D. Beutner, H. Harborth, Graceful labelings of nearly complete graphs, Results in Mathematics 41 (1) (2002) 34-39. doi:10.1007/BF03322754. URL https://doi.org/10.1007/BF03322754 
[26] T. H. Cormen, C. E. Leiserson, R. L. Rivest, C. Stein, Introduction to Algorithms, 3rd Edition, MIT Press, 2009. URL http://mitpress.mit.edu/books/introduction-algorithms

[27] Q. de Kang, Z. he Liang, Y. zhi Gao, G. hua Yang, On labeling of some graphs, J. Combin. Math. Combin. Comput. 22 (1996) 193-210.

[28] C. Morgan, Programming from specifications, $2^{\text {nd }}$ Edition, Prentice Hall International series in computer science, Prentice Hall, 1994.

[29] M. Horton, Graceful trees: Statistics and algorithms, 2003.
[30] Z. Nikoloski, N. Deo, F. Suraweera, Generation of graceful trees, in: In Proceedings of the 33 Southeastern International Conference on Combinatorics, Graph Theory and Computing.

[31] R. E. L. Aldred, B. D. Mckay, Graceful and harmonious labelings of trees, Bulletin of the institute of combinatorics and its applications 23 (1998) 69-72.

[32] R. Montgomery, A. Pokrovskiyy, and B. Sudakov, A proof of Ringel's Conjecture, arXiv:2001.02665v2 [math.CO] 23 Feb 2020.

\section{Creative Commons Attribution License 4.0 (Attribution 4.0 International, CC BY 4.0)}

This article is published under the terms of the Creative Commons Attribution License 4.0 https://creativecommons.org/licenses/by/4.0/deed.en US 